\title{
A design of fuzzy inference systems to predict tensile properties of as-cast alloy
}

\author{
He $\operatorname{Tan}^{1}$ (D) . Vladimir Tarasov ${ }^{1} \cdot$ Anders E. W. Jarfors ${ }^{1,2} \cdot$ Salem Seifeddine $e^{1,2}$
}

Received: 30 June 2020 / Accepted: 15 December 2020 / Published online: 1 February 2021

(C) The Author(s) 2021

\begin{abstract}
In this study, a design of Mamdani type fuzzy inference systems is presented to predict tensile properties of as-cast alloy. To improve manufacturing of light weight cast components, understanding of mechanical properties of cast components under load is important. The ability of deterministic models to predict the performance of a cast component is limited due to the uncertainty and imprecision in casting data. Mamdani type fuzzy inference systems are introduced as a promising solution. Compared to other artificial intelligence approaches, Mandani type fuzzy models allow for a better result interpretation. The fuzzy inference systems were designed from data and experts' knowledge and optimized using a genetic algorithm. The experts' knowledge was used to set up the values for the inference engine and initial values for the database parameters. The rule base was automatically generated from the data which were collected from casting and tensile testing experiments. A genetic algorithm with real-valued coding was used to optimize the database parameters. The quality of the constructed systems was evaluated by comparing predicted and actual tensile properties, including yield strength, Y.modulus, and ultimate tensile strength, of as-case alloy from two series of casting and tensile testing experimental data. The obtained results showed that the quality of the systems has satisfactory accuracy and is similar to or better than several machine learning methods. The evaluation results also demonstrated good reliability and stability of the approach.
\end{abstract}

Keywords Fuzzy logic system · Genetic algorithm · Genetic fuzzy system · Mechanical properties prediction · Lightweight cast components $\cdot \mathrm{Al}-\mathrm{Si}-\mathrm{Mg}$ alloy

\section{Introduction}

Casting is an important engineering area, which provides very effective methods to produce near net shape components and offers a great design freedom [12]. For light

\author{
He Tan \\ he.tan@ju.se \\ Vladimir Tarasov \\ vladimir.tarasov@ju.se \\ Anders E. W. Jarfors \\ anders.jarfors@ju.se \\ Salem Seifeddine \\ salem.seifeddine@ju.se
}

1 Department of Computer Science and Informatics, School of Engineering, Jönköping University, Box 1026, 55111 Jönköping, Sweden

2 Department of Materials and Manufacturing, School of Engineering, Jönköping University, Box 1026, 55111 Jönköping, Sweden weight cast components, the automotive industry is the largest user. ${ }^{1}$ This will be strongly affected by the transition from the use of combustion engines to electrical vehicles where light weight design and sustainability are likely to increase in importance. Designing for weight reduction can be made by material replacement or by a lighter design in similar materials. Most commonly this means less material to carry the load resulting in similar or higher levels of stress making it necessary to understand and to describe material behaviour on component level in detail to design for minimum warranty risk and maximum weight reduction. Most castings fail due to fatigue load but for automotive applications ductility requirements are equally important. The understanding of this behaviour is under extensive research for wrought materials, but cast materials, with many different large phases present, resulting in a great variation in mechanical properties, are poorly understood [13] and the spread in fatigue life as well as in ductility is significant [20].

\footnotetext{
${ }^{1}$ OICAs statistics of worldwide vehicle sales http://www.oica.net/ category/sales-statistics/
} 
The performance of a cast component depends on a number of factors. Deterministic models will be highly useful in a part design process as it will be distinct differences between different design and solutions. It must be pointed out, however, that other imperfections resulting from melt treatment and casting process will inherently display a stochastic behaviour. One such example is oxide films [3] being discrete particles of varying size formed during processing that will end up in discrete locations but not in a repeatable fashion. This will affect many different material properties, not only because of their amount but also through their orientation related to load direction.

In addition to this, there are a number of uncontrolled parameters that vary in a foundry, which will also result in variations in part quality and accuracy of a simulation [17]. These variations will limit the ability of a deterministic model to predict the actual outcome as well as necessitate management of the uncertainty. The quality of available data also affects prediction capabilities. In standards, such as for instance ASTM E8 and ASTM B557, there are options to use cast tensile bars or to take out tensile bars from a component. It is well known that the test bar dimensions affect performance but even more so the location in a component from where the test bars is taken.

Several artificial intelligence (AI) approaches, such as Bayesian theory (e.g. [18]), neural networks (e.g. [16]), and fuzzy logic (e.g. [1, 22]), have been proposed to deal with uncertainty and imprecision when predicting mechanical properties of cast and metallic components or relating mechanical properties to the parameters of the casting process. However, the current research has not fully addressed the mapping of mechanical properties of metallic materials to the casting process parameters.

The purpose of this paper is to contribute a new application of genetic fuzzy systems in the domain of modelling and prediction of mechanical properties of as-cast alloy for casting industry. The study advances the field by applying fuzzy logic models for prediction of several most important mechanical properties with the long term aim to predict fatigue life of as-cast alloy. In contrast to other AI approaches, such as neural networks or machine learning methods which mostly are a "black box", a fuzzy logic model is a highly interpretable grey-box model when Mamdani type fuzzy model is applied [9]. This makes fuzzy logic especially interesting for industrial applications in casting industry because transparency is often required by the stakeholders for the system to be used at a working place.

The main challenge of applying fuzzy logic based approaches is the manual and time consuming procedure for rule generation and membership parameter tuning. This paper proposes the use of genetic fuzzy systems (GFS) to address this challenge and further develops our approach in [22]. The application of the genetic algorithms (GA) to fuzzy systems is well-established $[5,6,10]$. GA learn or tune fuzzy inference system (FLS) by searching optimal solutions for the knowledge base (KB) parameters of a FLS with respect to the design criteria. In work [10] genetic fuzzy systems are grouped into different categories according to what parts of the $\mathrm{KB}$ are subject to learning or optimization using genetic algorithms. In this study membership functions (MF) were tuned using genetic algorithm with real value coding. The rule base was automatically learned from numerical experimental data. To reduce the search space and make the search process more efficient, the initial values for MF parameters are determined based on experts' knowledge.

The performance of the constructed FLS was examined for prediction of yield strength, Y.modulus, and ultimate tensile strength of as-cast Al-Si-Mg alloy in two series of data sets collected from casting and tensile testing experiments. The results showed high accuracy of prediction. When compared with several machine learning methods, the prediction accuracy of the constructed FLS is at the same level as or slightly better than the accuracy of the machine learning methods. In practice, the outcome of the study contributes a method that can be easily implemented and applied in casting industry to do predictive modelling, which allows for interpretation of the results.

The paper is structured as follows. Section 2 describes related work. Section 3 presents details of melt preparation and casting, results of tensile testing and data sets collected in the experiments. The approach to design, learn and tune FLS is described in Section 4. Section 5 presents the results of the evaluation of FLS. In Section 6 the reliability of the design and the stability of the generic tuning process are discussed. The comparison of the performance of the FLS and several machine learning methods is also presented in Section 6. The conclusions are drawn in Section 7.

\section{Related work}

Although the attempt to apply fuzzy logic in casting and metal industry is found to be limited, the studies have shown that fuzzy logic models of mechanical properties of die casts and metallic materials can achieve desired performance. The authors in [1] used fuzzy logic to predict surface roughness of die casting alloys after machining. The $\mathrm{Al}-11.3 \mathrm{Si}-2 \mathrm{Cu}-0.4 \mathrm{Fe}$ alloy was treated with different additives such as $\mathrm{Sr}, \mathrm{Bi}$, and $\mathrm{Sb}$ and workpieces were cast after that. A FLS was used to map the presence of additives and machining parameters to surface roughness with the error of 5.4\%. In the work [11] a fuzzy expert system was built to first optimize parameters of the milling process for a given objective, e.g. maximizing tool life, and then predict 
performance measures, including tool life, workpiece surface roughness, and three orthogonal components of cutting forces, for the selected milling parameters values. The milling parameters include milling orientation, cutter's helix angle, workpiece material hardness and coolant. The results showed the expert system was efficient for optimizing the hard-milling process and can provide desired predictions before start of actual process. The fuzzy logic model built in [25] predicted surface hardness of aluminium alloy with TiN (titanium nitride) coating with the help of four inputs: direct current power, substrate temperature, nitrogen flow rate and direct current bias voltage. The percentages of error and accuracy of the model were 6.09 and $96.142 \%$, respectively. In our previous work [22] two fuzzy logic models were constructed to map yield strength to the chemical composition and the rate of solidification of castings for two $\mathrm{Al}-\mathrm{Si}-\mathrm{Mg}$ alloys. The prediction errors of the models are $3.53 \%$ for the first and $3.19 \%$ for the second alloy.

Manual creation of fuzzy logic models serves the purpose well. However, when the volume of data underlying the model development increases, the manual creation of models becomes unfeasible. Several studies utilized artificial neural networks to learn and/or tune parameters of FLSs when they were built to predict mechanical properties and optimize casting process. In the work [8] an adaptive neuro-fuzzy inference system (ANFIS) was developed to identify the relationships between tensile strength and the structure parameters of gray cast iron, such as graphite flake size, microhardness of the matrix, amount of austenite dendrite and the eutectic cell count. The results were compared to the ones obtained by multiple linear regression, fuzzy regression and the generalized regression neural network. It is showed that ANFIS provides desired learning precision, but also good generalization for noised data compared to the regression methods. The work [19] describes the application of ANFIS and particle swarm optimization (PSO) method to optimize the parameters in processing of aluminium metal matrix composites, with a goal of minimizing the value of porosity and maximizing the value of strength and hardness. The parameters include mould temperature, mix time, impeller speed, powder temperature, cast temperature, particle volume percentage and particle size. The objective function was calculated by ANFIS and minimized by PSO. The system built by the authors can estimate hardness of a compo casting with the average testing error of 3.96 .

The work in [23] developed a system to predict the depth of cut in abrasive water jet machining process using fuzzy logic. The study compared three approaches to build the fuzzy logic system: 1) manually constructed the system; 2) optimized the data base and rule base of the system using GA; and 3) the system was evolved automatically using GA. The study showed that the average absolute percent deviation values in prediction for the approach 3 is 1.983 , which performed better than the approach 1 (2.689) and the approach 2 (4.377). The main focus of the aforementioned works is to apply fuzzy logic to model and predict tensile properties of as-cast alloy. To construct the fuzzy logic systems for predication, GA was applied to tune and optimize the shapes of membership functions, and rule base was generated by automatically examining the experimental data. The performance of the systems was compared with the performance of the systems manually constructed and with the performance of several machine learning methods and neural networks.

\section{Casting and tensile testing experiment}

The details of the experiment is depicted in this section. The experiment was carried out as the process presented in Fig 1. The data collected during this experimental phase were used to design, built and evaluate the FLS described in Section 4.

\subsection{Melt preparation and casting}

Seven $\mathrm{Al}-7 \% \mathrm{Si}-0.4 \% \mathrm{Mg}$ alloys, based on $\mathrm{A} 356$ master alloy, modified with approximately 200-250 ppm Sr and alloyed with various $\mathrm{Cu}$ concentrations were melt in a resistance furnace. Cylindrical rods (length $18 \mathrm{~cm}$, diameter 1 $\mathrm{cm})$ were thereafter cast in a $200{ }^{\circ} \mathrm{C}$ preheated permanent copper mould. Afterwards, the rods were inserted into the Bridgeman furnace, Fig. 2, at $710{ }^{\circ} \mathrm{C}$, remelted for $20 \mathrm{~min}$ and thereafter directionally solidified. The speed of the furnace during the passage of molten rod during the cooling channels determines the solidification rate of the samples; different microstructures can thereby be produced by changing the speed. Three different coarsenesses of the microstructure having Secondary Dendrite Arm Spacing, SDAS, of approximately 10,25 and $50 \mu \mathrm{m}$, were directionally solidified for the present investigation. Water cooling was used for high furnace speeds, $3 \mathrm{~mm} / \mathrm{s}$ and $0.3 \mathrm{~mm} / \mathrm{s}$ corresponding to SDAS of 10 and $25 \mu \mathrm{m}$ respectively, whereas no water cooling was used for the $0.03 \mathrm{~mm} / \mathrm{s}$ velocity that corresponds to SDAS of $50 \mu \mathrm{m}$; instead air was the cooling media.

\subsection{Tensile testing}

The experiment was performed according to the standard ASTM B557M. Tensile test bars with a gauge length of $50 \mathrm{~mm}$ and a diameter of $7 \mathrm{~mm}$ were machined from the directionally solidified rods. Tensile tests were performed at a constant strain rate of $0.5 \mathrm{~mm} / \mathrm{min}$ using a Zwick/Roell Z100 machine equipped with a $100 \mathrm{kN}$ load cell and a 
Fig. 1 The process of the casting and tensile testing experiment

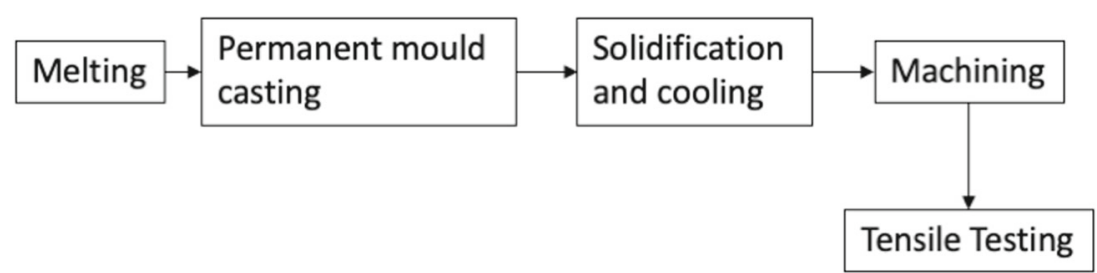

clip-on 20-mm gauge length extensometer. The samples were tested until fracture, using three tensile test bars for each condition. Since the proposed directional solidification technology has proven to deliver optimal tensile test results due to excellent feeding during solidification, revealing the potential of studied alloys, only samples that represents the potential of the alloy in term of tensile properties are presented.

\subsection{Experimental data}

In total two series of experiments were conducted. The first series was conducted on specimens cast with the alloy-Al-7\% Si- $0.4 \% \mathrm{Mg}-0.25 \% \mathrm{Fe}$ with variation of $\mathrm{Cu}$. The second series was based on alloy-Al-7\%Si- $0.4 \% \mathrm{Mg}$ with variation of $\mathrm{Si}$. In both series the solidification rate of the samples was varied as described above. Each experiment generated a data set on cast specimens that included variation of the chemical composition of cast alloys, i.e. percentage of $\mathrm{Cu}$ or $\mathrm{Si}$, and variation in the casting process, i.e. SDAS.

\section{Design of the Mamdani type fuzzy logic systems}

This section presents an approach to designing FLS with the help of genetic tuning knowledge base parameters and learning inference rules. The purpose of the created FLS is to predict several different tensile properties. The systems are based on a multiple-input-single-output Mamdani type fuzzy model. The inputs are the parameters of the process of

Fig. 2 The Bridgman furnace; directional solidification equipment

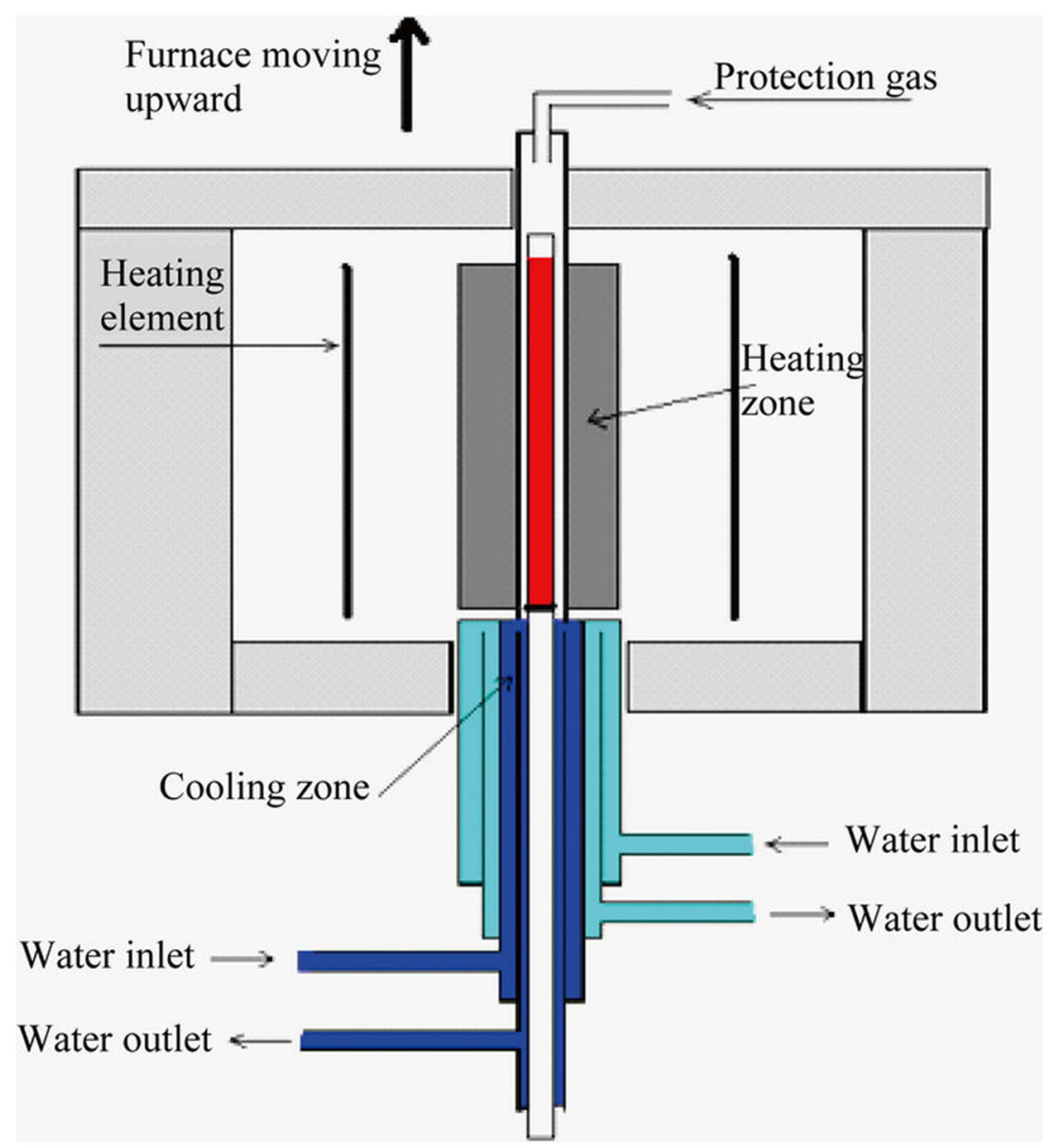


manufacturing as-cast alloys, i.e. the chemical composition of an alloy and the rate of solidification. The output is one of the tensile properties of the cast specimens. The approach to learn and tune the FLS is presented in Fig. 3. In the Mamdani type FLS, KB can be considered comprised of two components, a database (DB) and a rule base (RB). The DB contains the linguistic term sets and the membership functions. The RB is a collection of linguistic rules. The generation of the entire $\mathrm{KB}$ comprises two different processes: to create and tune $\mathrm{DB}$ and to derive RB. It starts with an initial definition of DB using experts' knowledge. During the genetic tuning process an error measure of the performance of the FLS is used as the fitness function to adjust the shapes of membership functions in the DB so that the performance of the system can be improved. When the DB definition is defined (in the initial step) or adjusted (during the tuning process), the RB is learned from training data set using the given DB definition. The approach is a combination of genetic DB tuning and RB learning from numerical examples. It does not belong to any of the categories defined in [10].

\subsection{Inference engine parameters and initial DB definition}

In this study seven membership functions were created for each linguistic variable during the initial DB design. The MF number was chosen to allow for the best coverage of the variable ranges extracted from the data sets. This MF number was also considered adequate for the nature of the problem being addressed by the domain experts. Moreover, previous studies in the area corroborated this choice by showing that the typical number of membership functions for a linguistic variable is between three and seven $[1$, $4,25]$, two and more than seven membership functions being a more rare case $[2,11]$, and finally the evaluation results presented in Section 6.1 confirmed this choice of the number of membership functions.

The type of the membership functions was chosen as Gaussian for all the input and output variables based on

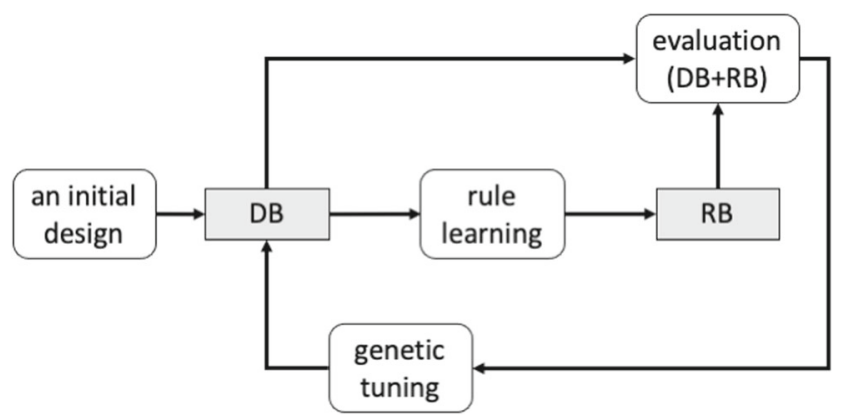

Fig. 3 The process of constructing FLS the initial check of MF types and discussion with the domain experts. In the beginning of the study, three types of MF were tested with the first data set. The types were Gaussian, generalized bell and triangular, including two variations of the shape of each type. The three types were compared by predicting yield strength (YS) with the manually constructed FLS (cf [22]), only the MF type being changed each run. Mean Absolute Percentage Error (MAPE) was computed using the test data. The results showed that the triangular type was the worst one in almost all the cases and the best one was either Gaussian or generalized bell. After that, the discussion of the MF types was conducted with the domain experts and they chose the Gaussian type as the most appropriate for the domain of casting and tensile testing.

The shapes of the membership function are randomly defined but following the rule: $\mu$ (mean) of the MF "high" can be any value in the variable range, but always higher than a $\mu$ (mean) of the MF "low", and the values for $\sigma$ (standard deviation) is randomly chosen from the variable range. The fuzzy inference rules were identified as $A N D$ rules. Centroid was used for defuzzification because of its wide acceptance and capability in giving more accurate results compared to the other methods $[1,11,25]$. The parameters are shown in Table 1.

\subsection{Learning RB}

For each FLS the rules are a set of two-input one-output inference rules. The rules have been identified as "and" rules (i.e. logical conjunction, which is denoted as \&) by a knowledge engineering expert as described in Section 4.1. The method of learning rules is adapted from a simple and straightforward method presented in [24]. The quality of the rules constructed by the learning method has been evaluated in [21]. In the experiment presented in [21], the rules generated with the given MF and using the learning method were identical to the manually created rules. The method consists of three steps:

1) Generate a rule for each input-output data pair, for example, $\left(x_{1}, x_{2} ; y\right)$ in training data:

Table 1 Initial DB definition and the inference engine parameters

\begin{tabular}{ll}
\hline Parameters & Value \\
\hline Number of MFs for & 7 \\
each input and output & \\
MFs' type & Gaussian \\
MFs' parameters $(\sigma$ and $\mu)$ & Random values \\
Defuzzification & Centroid \\
Antecedent connective & $A N D$ (min) \\
\hline
\end{tabular}


IF $x_{1}^{i}$ is $A$ and $x_{2}^{i}$ is $B$, THEN $y^{i}$ is $C$,

where $\mu_{A}\left(x_{1}^{i}\right), \mu_{B}\left(x_{2}^{i}\right)$, and $\mu_{C}\left(y^{i}\right)$ are membership functions with the maximum membership degree for each respective value.

For example, as shown in Fig. $4, x_{2}^{i}$ has degree 0.8 in "little", 0.2 in "very little", and 0 in other regions; therefore, $x_{2}^{i}$ is considered to be "little". In Fig. $4, x_{1}^{i}$ is considered to be "average", and $y^{i}$ is considered to be "average". The rule obtained from the input-output data pair is:

IF $x_{1}^{i}$ is average and $x_{2}^{i}$ is little, THEN $y^{i}$ is average.

2) Calculate a degree for each rule:

$$
D\left(\text { rule }^{i}\right)=\mu_{A}\left(x_{1}^{i}\right) \times \mu_{B}\left(x_{2}^{i}\right) \times \mu_{C}(y)^{i}
$$

For example, the degree for the rule above is 0.48 , since $D\left(\right.$ rule $\left.^{i}\right)=1 \times 0.8 \times 0.6=0.48$.

3) Generate the final list of rules

If there is more than one rule with the same antecedent, the rule that has the maximum degree is selected into the final list. For example,

Rule 1: IF $x_{1}^{i}$ is $A$ and $x_{2}^{i}$ is $B$, THEN $y^{i}$ is $C 1$, and the degree for rule 1 is 0.48 .

Rule 2: IF $x_{1}^{i}$ is $A$ and $x_{2}^{i}$ is $B$, THEN $y^{i}$ is $C 2$, and the degree of rule 2 is 0.52

Then, rule 2 is selected into the final list of the rules, since it has higher degree than rule 1 .

\subsection{Genetic DB tuning}

The shapes of MF affect the performance of FLS. In the initial design the value of $\sigma$ and $\mu$ are randomly given. In this process of genetic turning the shapes of MF are adjusted and evolve into an optimal solution. The fitness function is based on the performance of the FLS which is built using the MF represented in an individual and the rules constructed using the method described in Section 4.2.

\subsubsection{Encoding mechanism}

The operation of GA begins with a set of randomly generated individuals, called population. Each individual is a solution for the problem at hand. The MF have been identified as Gaussian functions. The goal is to find an optimal solution for the shapes of each MF in a FLS, i.e. the values for the parameters $\sigma$ and $\mu$. Each individual is represented in a real value encoded string,

$\left(\sigma_{M F_{x_{1}, 1}}, \mu_{M F_{x_{1}, 1}}, \ldots, \sigma_{M F_{x_{1}, n}}, \mu_{M F_{x_{1}, n}}\right.$,

$\sigma_{M F_{x_{2}, 1}}, \mu_{M F_{x_{2}, 1}}, \ldots, \sigma_{M F_{x_{2}, n}}, \mu_{M F_{x_{2}, n}}, \ldots$,

$\sigma_{M F_{x_{i}, 1}}, \mu_{M F_{x_{i}, 1}}, \ldots, \sigma_{M F_{x_{i}, n}}, \mu_{M F_{x_{i}, n}}, \ldots$,

$\left.\sigma_{M F_{y, 1}}, \mu_{M F_{y, 1}} \ldots, \sigma_{M F_{y, n}}, \mu_{M F_{y, n}}\right)$,

where $\left(x_{1}, x_{2}, \ldots, x_{i}\right)$ are input variables, $y$ is output variable. $n$ is the number of MFs for each variable. $\left(\sigma_{M F_{x_{i}, j}}, \mu_{M F_{x_{i}, j}}\right)$ are the standard deviation and mean for the $j$ th MF in the variable $x_{i}$, and $\mu_{M F_{x_{i}, m}}<\mu_{M F_{x_{i}, n}}$, if $m<n$. Figure 5 presents an example individual represented in such a real value encoded string.

\subsubsection{Initial population}

The initial population is randomly generated, while

$\mu_{M F_{x_{i}, i}}$ is randomly chosen from the range of the variable $x_{i},\left[\min _{x_{i}}, \max _{x_{i}}\right]$. To reduce the searching time, $\sigma_{M F_{x_{i}, i}}$ is randomly chosen from the range

$\left[\left(\max _{x_{i}}-\min _{x_{i}}\right) /(4 * n),\left(\max _{x_{i}}-\min _{x_{i}}\right) / n\right]$,

where $n$ is the number of MFs for variable.

\subsubsection{Fitness function}

The second step of GA is to determine how fit each individual in the population is. The fitness function calculates how well the whole FLS performs when it is applied to the set of training data. The prediction error of the FLS was measured using Mean Absolute Percentage Error (MAPE) [14]. It is computed by dividing the absolute difference of the predicted and measured values by the absolute value of the measured value and averaging this ratio over the data.

\subsubsection{Selection mechanism}

The individuals with higher fitness value have a better chance to be selected for reproduction. The individuals for
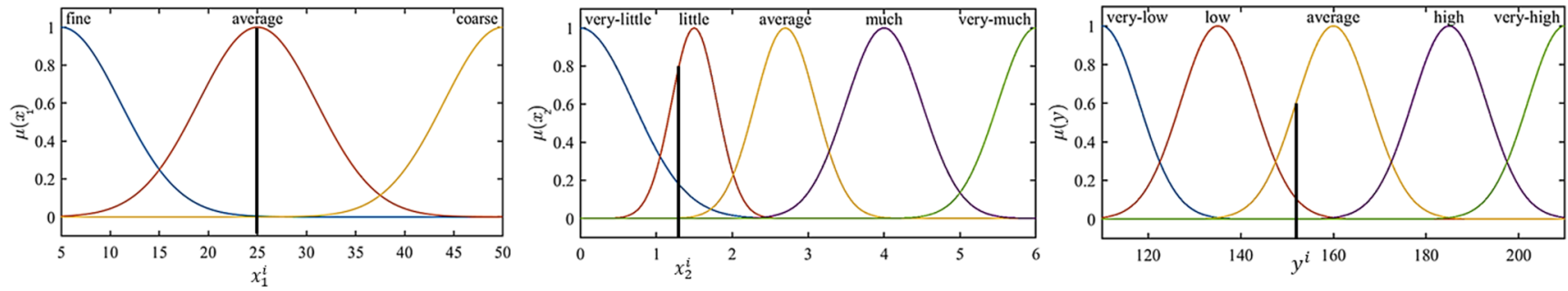

Fig. 4 The degrees of $x_{1}^{i}, x_{2}^{i}$ and $y^{i}$ in membership functions 
Fig. 5 An example of the real value encoded string for the MF parameters for all variables

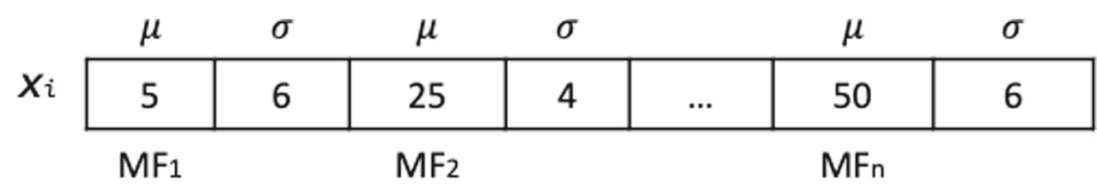

\begin{tabular}{c|c|c|c|c|c|c|}
\multicolumn{1}{c}{$\mu$} & $\sigma$ & $\mu$ & $\sigma$ & \multicolumn{2}{c}{$\mu$} & $\sigma$ \\
\hline \multicolumn{1}{c}{$y$} & 8 & 135 & 6 & $\ldots$ & 169 & 8 \\
\hline
\end{tabular}

reproduction are randomly selected based on probability. The probability is calculated as

$p\left(C_{i}\right)=\frac{f\left(C_{i}\right)}{\sum_{j=1}^{N} f\left(C_{i}\right)}$,

where $C_{i}$ represents individuals in a population, and $f\left(C_{i}\right)$ is the fitness value. An individual can be selected more than once.

\subsubsection{Crossover and mutation}

Crossover is the phase where the features of two parent individuals are combined to form an offspring, with the possibility that good individuals may generate better ones. The crossover operation used in the study is a simple discrete crossover. Let us assume that $C_{1}=\left(c_{1}^{1}, \ldots, c_{i}^{1}, \ldots, c_{n}^{1}\right)$ and $C_{2}=\left(c_{1}^{2}, \ldots, c_{i}^{2}, \ldots, c_{n}^{2}\right)$ are parent individuals to apply the crossover operations on them. An offstring, $O=$ $\left(o_{1}, \ldots, o_{i}, \ldots, o_{n}\right)$, is generated, where $o_{i}$ is a value randomly chosen from the set $\left\{c_{i}^{1}, c_{i}^{2}\right\}$.

The role of mutation in the operation in GA is to prevent the premature convergence of GA to suboptimal solutions. It is to arbitrarily change one or more components, genes, of the generated offspring, usually with a low random probability.

After a mutation, the order of parameter pairs $\left(\sigma_{M F_{i}}\right.$, $\left.\mu_{M F_{i}}\right)$ in the generated offstring, $\left(\sigma_{M F_{1}}, \mu_{M F_{1}}, \ldots, \sigma_{M F_{i}}\right.$, $\left.\mu_{M F_{i}}, \ldots, \sigma_{M F_{n}}, \mu_{M F_{n}}\right)$, is sorted, so that $\mu_{M F_{i}}<\mu_{M F_{j}}$, if $i<j$,

\subsubsection{Elitism}

To make sure that the best individuals always survive from one generation to the next, an elitist strategy is used: the top $10 \%$ individuals with the highest fitness values are always copied into the next generation.

\section{Evaluation of the FLS}

In this section, we present the evaluation results of the FLS constructed using the approach introduced in Section 4. As described in Section 3, the experiments provided two data sets. Each data set contains a set of input-output data pairs,

$\left\{\left(x_{1}^{1}, x_{2}^{1} ; y^{1}\right), \ldots,\left(x_{1}^{i}, x_{2}^{i} ; y^{i}\right), \ldots,\left(x_{1}^{n}, x_{2}^{n} ; y^{n}\right)\right\}$,

where $x_{1}$ represents SDAS, $x_{2}$ represents the percentage of $\mathrm{Cu}$ or $\mathrm{Si}$, and $y$ represents the output. The output is yield strength (YS), Y.modulus (E) or ultimate tesnile strength (UTS). The first data set, when $x_{2}$ represents the percentage of $\mathrm{Cu}$, contained 67 data points. The second data set, when $x_{2}$ represents the percentage of $\mathrm{Si}$, contained 52 data points. Each of the two data sets were divided into two parts, a training data set and a test data set. The training data sets were used to learn the FLSs. The prediction accuracy of the created fuzzy models were evaluated with the help of the test data sets. The performance of a FLS was calculated based on the prediction errors. It was measured using Mean Absolute Percentage Error (MAPE).

When dividing each of the two data sets the test data set included one data point for each unique pair of $\left(x_{1}^{1}, x_{2}^{1}\right)$ value if it is applicable. The rest data points were assigned to the training data set. The first data set was divided into a training data set with 46 data points and a test data set with 21 data points. The second data set was divided into a training data set with 36 data points and a test data set with 16 data points.

When building a FLS, the population size for GA is 100 , the mating probability is 0.8 and the mutation probability is 0.1 . The program stops when the number of generations reaches 100 . The learning approach was implemented using SciPy, ${ }^{2}$ which is an open source software for scientific computing in Python, and the FLSs were constructed using a fuzzy logic toolbox ${ }^{3}$ for SciPy.

Tables 2 and 3 depict the evaluation results for the FLS that were constructed based on the individuals with the best fitness value in the 100th generation. The first column in each table contains the designation of a casting component from the test data sets. Both tables show the percentage of $\mathrm{Cu} / \mathrm{Si}$ and SDAS, which are the inputs to the FLSs, the measured value (indicated by " $\mathrm{M}$ " in the tables) and the

\footnotetext{
${ }^{2}$ https://www.scipy.org/

${ }^{3}$ https://pythonhosted.org/scikit-fuzzy/
} 
Table 2 Evaluation of the model accuracy for the first test data set, Al-SI-Mg with $\mathrm{Cu}$ variation

\begin{tabular}{|c|c|c|c|c|c|c|c|c|c|c|c|}
\hline Sample & $\mathrm{Cu}$ & SDAS & YS (M) & YS (P) & Err (\%) & $\mathrm{E}(\mathrm{M})$ & $\mathrm{E}(\mathrm{P})$ & Err (\%) & UTS (M) & UTS (P) & $\operatorname{Err}(\%)$ \\
\hline AA13 & 0 & 10 & 125.44 & 123.09 & -1.87 & 68053.18 & 66461.79 & -2.34 & 246.66 & 246.89 & 0.09 \\
\hline AA23 & 0 & 20 & 125.51 & 123.24 & -1.81 & 61137.19 & 62389.15 & 2.05 & 224.65 & 220.34 & -1.92 \\
\hline AA33 & 0 & 50 & 113.06 & 121.99 & 7.9 & 65820.27 & 62740.63 & -4.68 & 170.17 & 174.74 & 2.69 \\
\hline AB13 & 0.6 & 10 & 133.59 & 136.18 & 1.94 & 68849.19 & 68384.34 & -0.68 & 275.56 & 272.15 & -1.24 \\
\hline AB22 & 0.6 & 20 & 135.94 & 136.18 & 0.17 & 63353.80 & 64181.39 & 1.31 & 258.16 & 244.72 & -5.2 \\
\hline AB32 & 0.6 & 50 & 117.57 & 124.17 & 5.61 & 65320.13 & 64346.50 & -1.49 & 191.18 & 198.80 & 3.99 \\
\hline AC11 & 1 & 10 & 144.96 & 142.53 & -1.67 & 70537.16 & 69113.45 & -2.02 & 298.82 & 293.54 & -1.77 \\
\hline $\mathrm{AC} 21$ & 1 & 20 & 145.11 & 142.49 & -1.81 & 66074.29 & 65691.42 & -0.58 & 282.12 & 270.29 & -4.19 \\
\hline AC31 & 1 & 50 & 129.44 & 128.32 & -0.86 & 68445.27 & 65530.16 & -4.26 & 212.12 & 213.88 & 0.83 \\
\hline AD13 & 1.5 & 10 & 144.20 & 147.93 & 2.58 & 69389.37 & 70121.59 & 1.06 & 283.17 & 301.53 & 6.48 \\
\hline $\mathrm{AD} 23$ & 1.5 & 20 & 157.29 & 153.55 & -2.38 & 67081.33 & 68165.15 & 1.62 & 289.58 & 280.60 & -3.1 \\
\hline AD33 & 1.5 & 50 & 139.01 & 141.69 & 1.93 & 66898.05 & 67518.48 & 0.93 & 220.37 & 217.78 & -1.18 \\
\hline AE12 & 1.7 & 10 & 151.14 & 151.22 & 0.05 & 71186.23 & 70568.72 & -0.87 & 275.17 & 302.06 & 9.77 \\
\hline AE32 & 1.7 & 50 & 141.50 & 145.75 & 3.01 & 66363.42 & 67993.96 & 2.46 & 225.54 & 219.36 & -2.74 \\
\hline AF31 & 2.5 & 50 & 152.20 & 154.71 & 1.65 & 69039.02 & 69734.74 & 1.01 & 228.57 & 232.54 & 1.73 \\
\hline AG14 & 3.5 & 10 & 166.83 & 166.02 & -0.49 & 72598.10 & 72220.83 & -0.52 & 322.38 & 301.93 & -6.34 \\
\hline AG23 & 3.5 & 20 & 173.85 & 166.17 & -4.42 & 71660.78 & 72649.50 & 1.38 & 301.81 & 301.07 & -0.25 \\
\hline AG33 & 3.5 & 50 & 170.86 & 166.02 & -2.84 & 70124.57 & 69903.67 & -0.32 & 224.98 & 235.13 & 4.51 \\
\hline AI12 & 5.5 & 10 & 194.70 & 194.01 & -0.36 & 73289.52 & 70630.34 & -3.63 & 354.97 & 316.13 & -10.94 \\
\hline AI23 & 5.5 & 20 & 202.88 & 202.39 & -0.24 & 69693.16 & 69526.65 & -0.24 & 276.33 & 257.62 & -6.77 \\
\hline $\mathrm{AI} 32$ & 5.5 & 50 & 189.74 & 178.89 & -5.71 & 70454.65 & 69903.76 & -0.78 & 215.14 & 217.50 & 1.09 \\
\hline MAPE & & & & & 2.35 & & & 1.63 & & & 3.66 \\
\hline
\end{tabular}

Table 3 Evaluation of the model accuracy for the second test data set, Al-Si-Mg with Si variation

\begin{tabular}{|c|c|c|c|c|c|c|c|c|c|c|c|}
\hline Sample & $\mathrm{Si}$ & SDAS & YS (M) & YS (P) & Err $(\%)$ & $\mathrm{E}(\mathrm{M})$ & $\mathrm{E}(\mathrm{P})$ & $\operatorname{Err}(\%)$ & UTS (M) & UTS (P) & $\operatorname{Err}(\%)$ \\
\hline AIA13 & 7 & 10 & 125.44 & 123.19 & -1.79 & 68053.18 & 65436.14 & -3.84 & 246.66 & 245.02 & -0.66 \\
\hline AIA23 & 7 & 20 & 125.51 & 121.42 & -3.26 & 61137.19 & 61982.34 & 1.38 & 224.65 & 223.09 & -0.7 \\
\hline AIA33 & 7 & 50 & 113.06 & 120.34 & 6.44 & 65820.27 & 61493.13 & -6.57 & 170.17 & 171.28 & 0.65 \\
\hline AIB13 & 10 & 10 & 161.20 & 157.80 & -2.11 & 71496.53 & 72919.93 & 1.97 & 303.81 & 295.44 & -2.76 \\
\hline AIB23 & 10 & 20 & 132.35 & 132.05 & -0.23 & 68996.61 & 68929.34 & -0.09 & 228.71 & 226.32 & -1.04 \\
\hline AIB33 & 10 & 50 & 108.67 & 110.09 & 1.3 & 61077.64 & 60144.92 & 1.52 & 165.42 & 161.08 & -2.63 \\
\hline AIC13 & 11.5 & 10 & 168.94 & 163.72 & -3.09 & 78933.58 & 72612.32 & -8.00 & 313.56 & 321.80 & 2.63 \\
\hline AIC33 & 11.5 & 50 & 115.77 & 111.30 & -3.86 & 65749.59 & 66300.78 & 0.84 & 158.93 & 157.70 & -0.77 \\
\hline AID13 & 12.5 & 10 & 180.14 & 181.48 & 0.74 & 75064.49 & 73543.13 & -2.03 & 325.98 & 325.67 & -0.09 \\
\hline AID33 & 12.5 & 50 & 110.40 & 110.71 & 0.28 & 69141.32 & 66410.36 & -3.94 & 160.89 & 157.30 & -2.23 \\
\hline AIE13 & 13 & 10 & 176.33 & 186.13 & 5.56 & 73165.56 & 74321.28 & 1.58 & 319.83 & 325.70 & 1.84 \\
\hline AIE23 & 13 & 20 & 136.41 & 132.09 & -3.17 & 74301.18 & 73121.68 & -1.58 & 225.42 & 226.32 & 0.4 \\
\hline AIE33 & 13 & 50 & 110.99 & 111.19 & 0.18 & 69037.38 & 66608.43 & -3.51 & 157.68 & 157.31 & -0.24 \\
\hline AIF13 & 14.5 & 10 & 176.03 & 187.95 & 6.77 & 75496.23 & 76462.71 & 1.28 & 319.94 & 325.63 & 1.78 \\
\hline AIF23 & 14.5 & 20 & 128.38 & 132.06 & 2.86 & 71551.20 & 69795.37 & -2.45 & 232.77 & 226.32 & -2.77 \\
\hline AIF33 & 14.5 & 50 & 118.65 & 118.24 & -0.35 & 73834.07 & 75996.94 & 2.92 & 174.42 & 169.73 & -2.69 \\
\hline МАPE & & & & & 2.62 & & & 2.72 & & & 1.49 \\
\hline
\end{tabular}


predicted value by the FLSs (indicated by "P") of YS, E and UTS, and a (signed) percentage error (marked by "\%") of each pair of the predicted and measured values of the tensile properties. The performance of a FLS was measured using MAPE. It is given at the bottom of each table. It is $2.35 \%$ when predicting YS, $1.63 \%$ when predicting E, and $3.66 \%$ when predicting UTS for the first fuzzy logic model. For the second model, MAPE is $2.62 \%$ when predicting YS, $2.72 \%$ when predicting E, and $1.49 \%$ when predicting UTS.
The related work reports the prediction accuracy of the fuzzy logic based systems that is similar to the prediction errors of the constructed fuzzy logic systems. The prediction errors were also discussed with the domain experts, who considered the error level as acceptable. While the performance of the fuzzy logic systems is good, it is worth noting that this work is one of the first steps on modelling of yield strength of as-cast alloys. Small data sets were used to create the models. The prediction accuracy may be different
Fig. 6 Evolution of MAPE across 100 generations in the evaluation when the first data set (Al-Si-Mg with $\mathrm{Cu}$ variation) was used. The $\mathrm{x}$-axis is the number of generation. The $y$-axis is the MAPE value. '\#MF' states the number of membership functions. a shows the prediction results for YS. b shows the prediction results for E. c shows the prediction results for UTS

\section{(a) Predition results for YS}

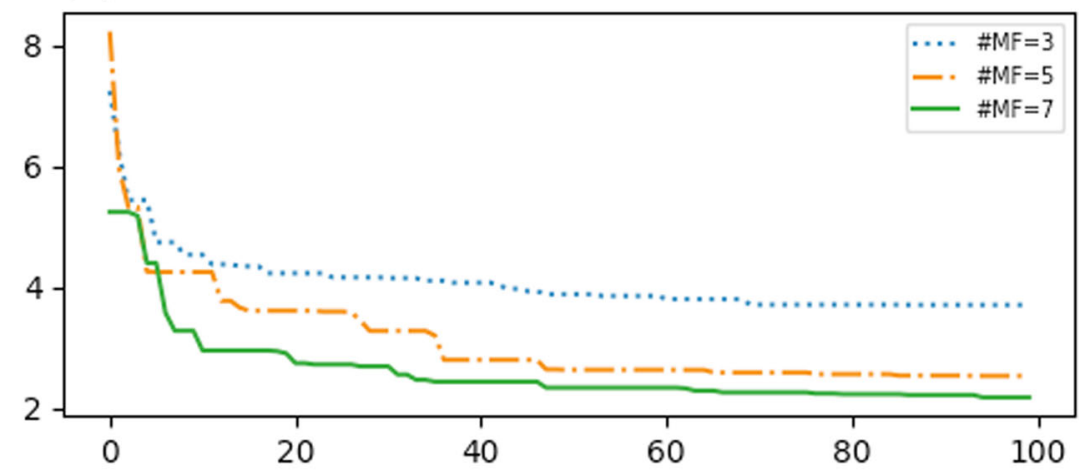

(b) Predition results for $\mathrm{E}$

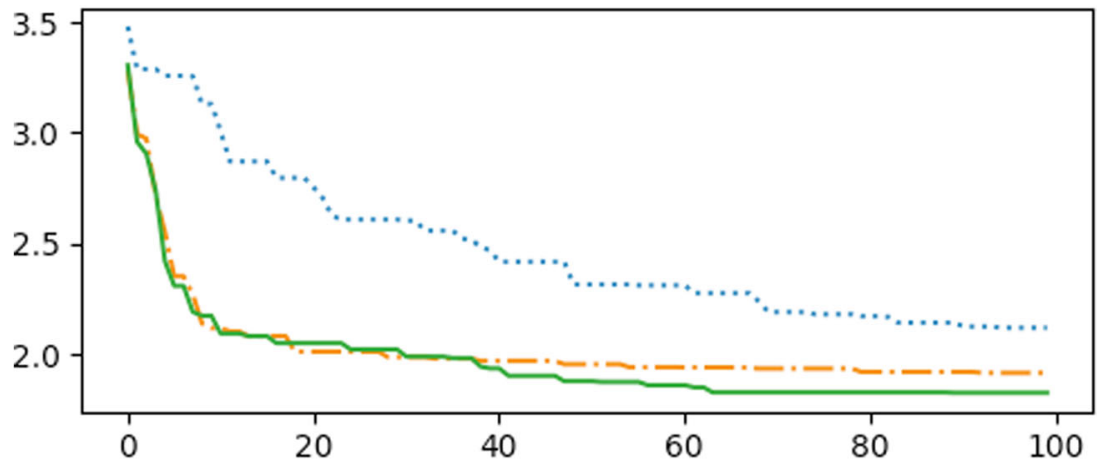

(c) Predition results for UTS

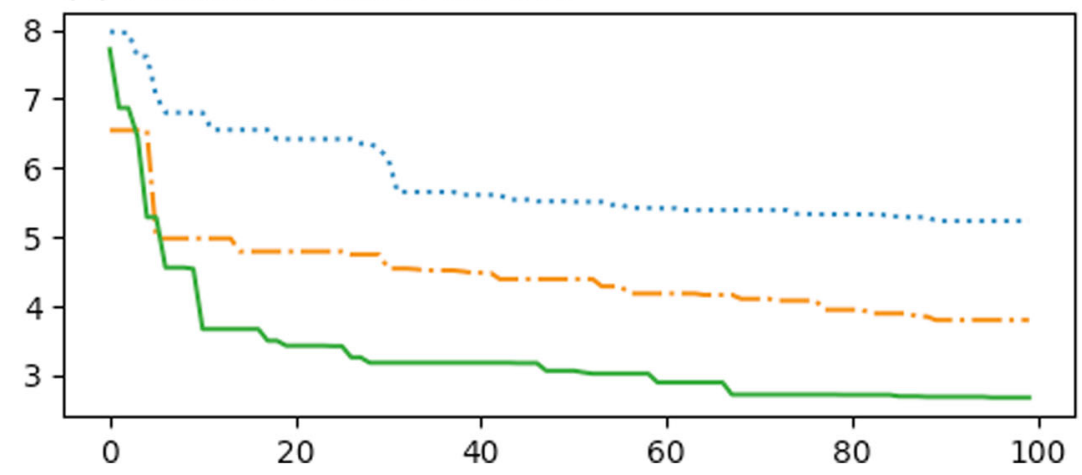


if bigger data sets are used for the modelling. Moreover, the procedure of data collection at a particular lab or foundry could affect the performance of the fuzzy logic models due to variations in measurement accuracy or unaccounted differences in the cast process.

\section{Discussion}

This section first discusses the reliability of the approach to design FLS, and then the stability of the FLS constructed using the approach. The discussion is supported by the evaluation results of the features. Secondly, the comparison is presented of the performance of the constructed FLS with several other methods of predictive modelling.

\subsection{Reliability and stability}

Since the study has shown that the typical number of membership functions for a variable is between three and seven $([1,4,25])$, seven was chosen for the number of the MFs for variables in the initial design to have the best coverage. The evaluation results presented in Figs. 6 and 7 confirmed this design decision. The figures show the
Fig. 7 Evolution of MAPE across 100 generations in the evaluation when the second data set (Al-Si-Mg with $\mathrm{Si}$ variation) was used. The $\mathrm{x}$-axis is the number of generation. The $y$-axis is the MAPE value. '\#MF' states the number of membership functions. a shows the prediction results for YS. b shows the prediction results for E. c shows the prediction results for UTS

\section{(a) Predition results for YS}

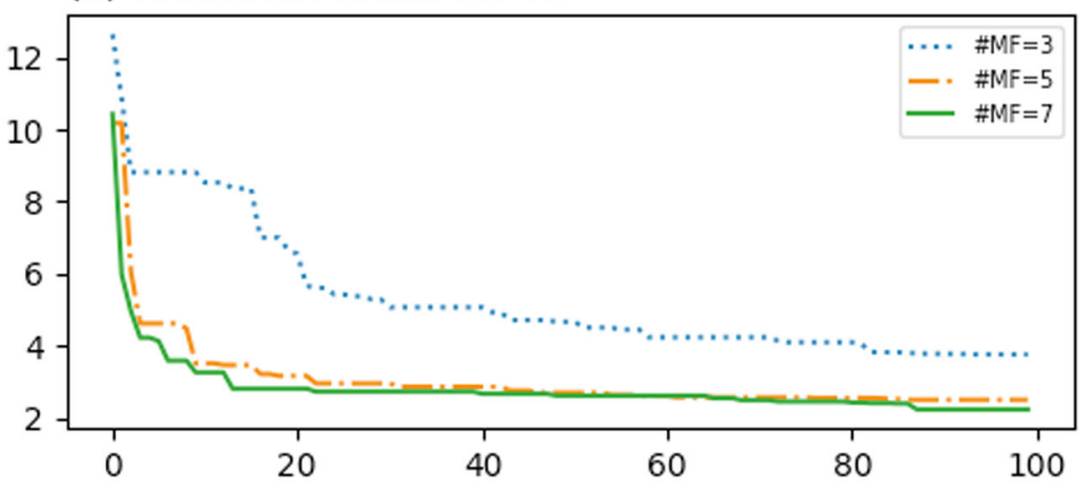

(b) Predition results for $\mathrm{E}$

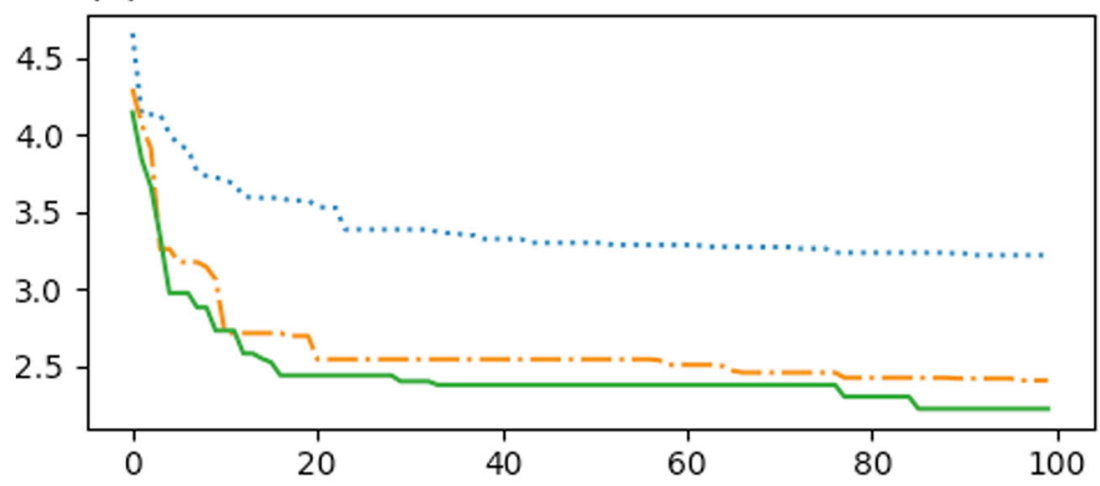

(c) Predition results for UTS

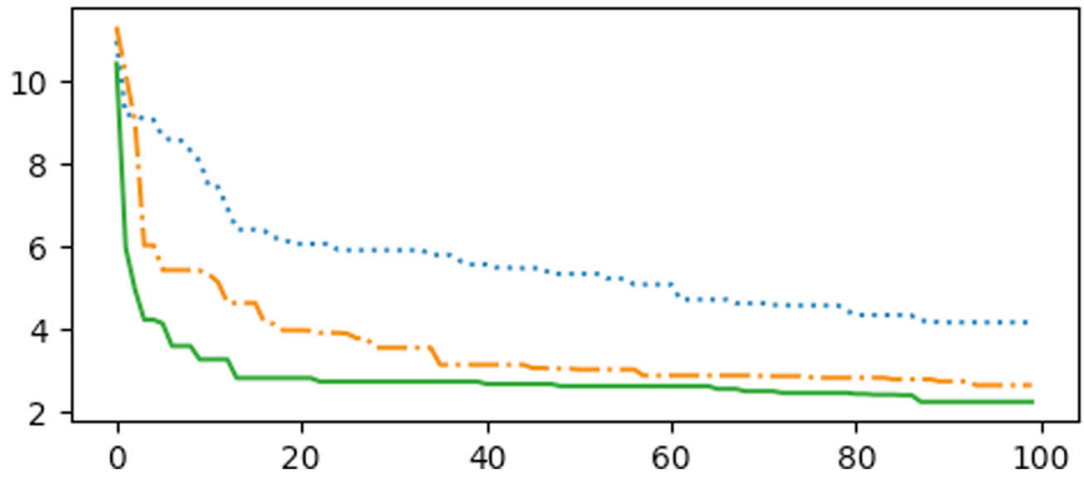


evolution of individuals with the best fitness value in the population across 100 generations when the number of MF were chosen as three, five or seven in the evaluations for the two data set. In both of the evaluations the genetic tuning converges to a solution in less than 100 generations in all the cases. The performance of the constructed FLS with seven MF is the best one for predicting all the three tensile properties in both of the evaluations. The results show that in most of the cases the performance of the constructed FLS with five or seven MF is very close, and much better than the FLS with three MF. When the cost of computational complexity is an important design consideration besides the accuracy of system performance, five MF can also be a choice.

GA are a stochastic optimization algorithm. It gives different results at different runs. Table 4 presents a stability of the method in three runs. The first column gives the name of the mechanical property to be predicted. The second, third and fourth columns present the performance (i.e. MAPE value) of the FLS in the three runs. The last three columns are the mean, standard deviation (SD) and coefficient variation (CV) of the MAPE values. It shows that the variance between the performance of the constructed FLSs in the three runs is very small.

\subsection{Comparison with other predictive models}

To evaluate the performance of the constructed FLS, they were compared with several machine learning methods. To this end, a number of parametric and nonparametric regression models as well as neural networks were constructed and tested with both data sets. The experimental data were divided into training and test sets as described in Section 5. The Matlab Statistics and Machine Learning Toolbox was used to implement the predictive models, which were created for the tensile properties YS, E, and UTS. The results are summarized in Table 5, which depicts MAPE for each of the modelling approaches (the table only shows the best result for the regression modelling and neural networks).

Table 4 Evaluation results in 3 runs for the two data sets

\begin{tabular}{|c|c|c|c|c|c|c|}
\hline MP & $\# 1$ & $\# 2$ & $\# 3$ & Mean & $\mathrm{SD}$ & $\mathrm{CV}$ \\
\hline \multicolumn{7}{|c|}{ The first data set } \\
\hline YS & 2.35 & 2.77 & 2.48 & 2.53 & 0.176 & 0.070 \\
\hline $\mathrm{E}$ & 1.63 & 1.64 & 1.63 & 1.63 & 0.005 & 0.003 \\
\hline UTS & 3.66 & 3.77 & 3.88 & 3.77 & 0.090 & 0.024 \\
\hline \multicolumn{7}{|c|}{ The second data set } \\
\hline YS & 2.62 & 2.80 & 2.99 & 2.80 & 0.151 & 0.054 \\
\hline $\mathrm{E}$ & 2.72 & 3.05 & 2.89 & 2.89 & 0.135 & 0.047 \\
\hline UTS & 1.49 & 1.90 & 1.52 & 1.64 & 0.187 & 0.114 \\
\hline
\end{tabular}

The following regression models were used to predict the tensile properties:

- Multivariate linear and quadratic regression,

- Support Vector Machine regression with linear and Gaussian kernels,

- Binary regression decision trees,

- Gaussian process regression.

As to neural networks, feed-forward regression networks were used with different training functions and one hidden layer of the default size (10 neurons). Part of the training data set was reserved for validation to evaluate performance of the network during the training. Each model was retrained 30 times because the network performance may change with each training run due to the differences in the initialisation of network parameters and division into training and validation sets. The mean MAPE was computed for the series of the runs to get more accurate results on the network performance.

The utilized training functions were:

- Levenberg-Marquardt backpropagation (the validation set size was $5 \%$ ),

- Bayesian Regularization (validation data are not used by this function),

- Scaled Conjugate Gradient (the validation set size was 5\%).

The results show that the prediction accuracy of the developed GFS is slightly better or comparable to the accuracy of the multiple regression models and regression feedforward networks with one hidden layer. The predictive modelling for the first data set demonstrates that for the property YS, the best MAPE is produced by regression modelling, while for E and UTS the GFS delivers the best result. For the second data set, the GFS gives the smallest MAPE for all the three tensile properties. However, the difference in the results is relatively small.

The regression neural networks demonstrated stable performance only with one training function, Bayesian

Table 5 Comparison of the genetic fuzzy systems with machine learning methods

\begin{tabular}{llll}
\hline Tensile Property & YS & E & UTS \\
\hline The first data set & & & \\
Genetic fuzzy system & 2.35 & 1.63 & 3.66 \\
Best regression model & 1.85 & 1.65 & 3.89 \\
Best neural network & 2.22 & 2.18 & 3.85 \\
The second data set & & & \\
Genetic fuzzy system & 2.62 & 2.72 & 1.49 \\
Best regression model & 2.77 & 2.48 & 2.01 \\
Best neural network & 2.84 & 2.92 & 2.20 \\
\hline
\end{tabular}


Regularization. The reason for this is that this training method is generally recommended for small and noisy data sets and the two data sets described in the paper are relatively small. The second training method, LevenbergMarquardt backpropagation, which is used by default in Matlab, gave modelling accuracy varying from run to run because it is intended for big enough data sets.

\section{Conclusions}

The presented work contributes a new application of genetic fuzzy systems in the field of modelling and prediction of mechanical properties for casting industry. In the approach, experts' knowledge was used to initialize the design of the FLS, the rule bases were automatically learned from the experimental data, and genetic algorithms were employed to tune and optimize the membership functions parameters. The proposed fuzzy logic systems are designed to model several of the most important mechanical properties, given the coarseness of microstructure and the percentage of $\mathrm{Cu} / \mathrm{Si}$ in the cast alloy. The evaluation of the constructed FLS showed high accuracy of prediction. The performance of constructed fuzzy logic systems is at the same level or slightly better than the accuracy of the fuzzy logic systems reviewed in Section 2. The evaluation results also demonstrated that the reliability and stability of the approach to construct the designed FLS is good.

This paper has presented a study to address the lack of understanding of mechanical behaviour of light weight cast components by applying fuzzy logic systems. Aluminium alloys have been used to replace steels, for example, in automotive parts to maintain the effectiveness of an engine vehicle. This is important to meet the demands of environmental sustainability. However, the mechanical behaviour of as-cast alloy is poorly understood. Due to uncertainty and imprecision in casting data, deterministic models have limits in predicting the mechanical properties. There is an obvious need to develop stochastic models to tackle this problem. As discussed in Section 1, the main advantage of a Mamdani type fuzzy logic system over machine learning methods is that the former provides a highly interpretable model. Having interpretable models that can be inspected and comprehended is important for industrial applications.

We see several future directions of research related to this work: (1) Elongation and elongation to failure are another two important mechanical properties highly related to fatigue life of aluminium alloys. It is even more difficult to capture the correlation function between fatigue life and these two properties. We would like to further investigate the use of fuzzy logic models to handle this problem. (2) Recently, there has been a surge of work on interpretability and explainability of neural networks in the area of deep learning (e.g. [7, 15]). We would like to investigate the advancement in the area and apply interpretable neural networks to address the challenges in prediction of mechanical properties. (3) Through the cooperation with industrial stakeholders, more extensive laboratory test data are expected to be obtained for fuzzy logic modelling and evaluation in the future.

Authors' contributions He Tan designed and implemented the methods, and analysed the results. Vladimir Tarasov helped verify the methods and results. Salem Seifeddine designed and performed the tensile testing and collected the data. Anders E.W. Jarfors helped supervise the project. All authors discussed the results and contributed to the final manuscript.

Funding Open Access funding provided by Jönköping University. The work presented in this paper was funded by the Knowledge Foundation in Sweden, grant KKS-20170066.

Data availability The data that support the findings of this study are available on request from the corresponding author.

Code availability The code that support the findings of this study are available on request from the corresponding author.

\section{Compliance with ethical standards}

Conflict of interest The authors declare that they have no conflict of interest.

Open Access This article is licensed under a Creative Commons Attribution 4.0 International License, which permits use, sharing, adaptation, distribution and reproduction in any medium or format, as long as you give appropriate credit to the original author(s) and the source, provide a link to the Creative Commons licence, and indicate if changes were made. The images or other third party material in this article are included in the article's Creative Commons licence, unless indicated otherwise in a credit line to the material. If material is not included in the article's Creative Commons licence and your intended use is not permitted by statutory regulation or exceeds the permitted use, you will need to obtain permission directly from the copyright holder. To view a copy of this licence, visit http:// creativecommonshorg/licenses/by/4.0/.

\section{References}

1. Barzani MM, Zalnezhad E, Sarhan AA, Farahany S, Ramesh S (2015) Fuzzy logic based model for predicting surface roughness of machined $\mathrm{Al}-\mathrm{Si}-\mathrm{Cu}-\mathrm{Fe}$ die casting alloy using different additives-turning. Measurement 61(Supplement C):150-161

2. Boukezzi L, Bessissa L, Boubakeur A, Mahi D (2017) Neural networks and fuzzy logic approaches to predict mechanical properties of XLPE insulation cables under thermal aging. Neural Comput Applic 28(11):3557-3570

3. Campbell J (2011) Complete casting handbook: metal casting processes, techniques and design. Butterworth-Heinemann, Oxford

4. Chiang KT, Liu NM, Chou CC (2008) Machining parameters optimization on the die casting process of magnesium alloy using the grey-based fuzzy algorithm. Int J Adv Manuf Technol 38(3):229-237 
5. Cordón O (2011) A historical review of evolutionary learning methods for Mamdani-type fuzzy rule-based systems: designing interpretable genetic fuzzy systems. Int $\mathrm{J}$ Approx Reason 52(6):894-913

6. Cordón O, Gomide F, Herrera F, Hoffmann F, Magdalena L (2004) Ten years of genetic fuzzy systems: current framework and new trends. Fuzzy Sets Syst 141(1):5-31

7. Gilpin LH, Bau D, Yuan BZ, Bajwa A, Specter M, Kagal L (2018) Explaining explanations: an overview of interpretability of machine learning. In: 2018 IEEE 5th International Conference on data science and advanced analytics (DSAA). IEEE, pp 80-89

8. Hancheng Q, Bocai X, Shangzheng L, Fagen W (2002) Fuzzy neural network modeling of material properties. J Mater Process Technol 122(2-3):196-200

9. Hellendoorn H, Driankov D (2012) Fuzzy model identification: selected approaches. Springer Science \& Business Media, New York

10. Herrera F (2008) Genetic fuzzy systems: taxonomy, current research trends and prospects. Evol Intel 1(1):27-46

11. Iqbal A, He N, Li L, Dar NU (2007) A fuzzy expert system for optimizing parameters and predicting performance measures in hard-milling process. Expert Syst Appl 32(4):1020-1027

12. Jarfors AEW, Bejhem M, Carlsson T, Eliasson A, Keife H, Nicolescu CM, Rundqvist B (2010) Tillverkningsteknologi, 4th edn. Lund, Studentlitteratur

13. Krupp U (2007) Fatigue crack propagation in metals and alloys: microstructural aspects and modelling concepts, Wiley, New York

14. de Myttenaere A, Golden B, Grand BL, Rossi F (2016) Mean absolute percentage error for regression models. Neurocomputing 192:38-48. Advances in artificial neural networks, machine learning and computational intelligence

15. Olah C, Satyanarayan A, Johnson I, Carter S, Schubert L, Ye K, Mordvintsev A (2018) The building blocks of interpretability. Distill 3(3):e10

16. Ozerdem MS, Kolukisa S (2009) Artificial neural network approach to predict the mechanical properties of $\mathrm{Cu}-\mathrm{Sn}-\mathrm{Pb}-\mathrm{Zn}-\mathrm{Ni}$ cast alloys. Mater Des 30(3):764-769
17. Raza M, Svenningsson R, Irwin M, Fägerström B, Jarfors AE (2017) Effects of process related variations on fillablity simulation of thin-walled IN718 structures. Springer, Berlin

18. Santos I, Nieves J, Penya YK, Bringas PG (2009) Machinelearning-based mechanical properties prediction in foundry production. In: 2009 ICCAS-SICE. IEEE, pp 4536-4541

19. Shabani MO, Rahimipour MR, Tofigh AA, Davami P (2015) Refined microstructure of compo cast nanocomposites: the performance of combined neuro-computing, fuzzy logic and particle swarm techniques. Neural Comput and Applic 26(4):899909

20. Shirani M, Härkegård G (2012) Damage tolerant design of cast components based on defects detected by 3D X-ray computed tomography. Int J Fatigue 41(Supplement C):188-198. Fatigue Design \& Material Defects

21. Tan H, Tarasov V, Jarfors AE, Seifeddine S (2019) Fuzzy logic based modelling of cast component properties. IFACPapersOnLine 52(13):1132-1137

22. Tarasov V, Tan H, Jarfors AE, Seifeddine S (2019) Fuzzy logicbased modelling of yield strength of as-cast A356 alloy. Neural Comput Applic, 1-12

23. Vundavilli PR, Parappagoudar M, Kodali SP, Benguluri S (2012) Fuzzy logic-based expert system for prediction of depth of cut in abrasive water jet machining process. Knowl-Based Syst 27:456464

24. Wang LX, Mendel JM (1992) Generating fuzzy rules by learning from examples. IEEE Trans Syst Man Cybern 22(6):14141427

25. Zalnezhad E, Sarhan AAD, Hamdi M (2013) A fuzzy logic based model to predict surface hardness of thin film TiN coating on aerospace AL7075-T6 alloy. Int J Adv Manuf Technol 68(1):415423

Publisher's note Springer Nature remains neutral with regard to jurisdictional claims in published maps and institutional affiliations. 